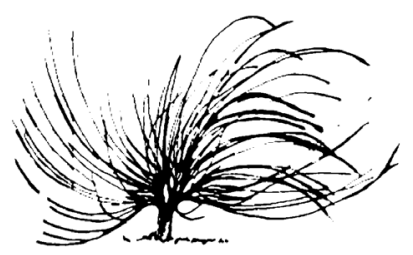

\title{
Análisis de los niveles de actividad física de los estudiantes, el tipo de contenidos de la lección y la interacción del profesor durante la clase de educación física en escuelas públicas
}

\author{
Carlos Álvarez Bogantes ${ }^{1}$ \\ Universidad Nacional \\ Costa Rica \\ ceab.03@gmail.com
}

\begin{abstract}
Resumen
El objetivo de la investigación fue describir los niveles de actividad física (AF) de los estudiantes de escuelas primarias de zona urbana y semiurbana, durante las clases de educación física, en el contexto de la lección de educación física $(\mathrm{EF})$ y las interacciones del maestro. Este es un estudio descriptivo-comparativo transversal de observación de 360 estudiantes durante las clases de I ciclo en escuelas de primaria públicas. Para evaluar estas variables, se utilizó el Sistema para Observar la Instrucción de la Aptitud Física (SOFIT). Como resultado, los estudiantes realizan menos actividad física que el promedio recomendado por la literatura. Solamente un $30.2 \%$ y un $9 \%$ de las lecciones de EF correspondieron a actividades de moderada (AMI) y vigorosa $(\mathrm{AV})$ intensidad, respectivamente. Las mujeres presentaron niveles más bajos de AF que los hombres y las escuelas en áreas urbanas no presentaron mostraron más
\end{abstract}

\section{(c) (1) $(\$)$}

Recibido: 7 de noviembre de 2017. Aprobado: 24 de setiembre de 2018 .

http://dx.doi.org/10.15359/rep.13-2.4

1. Promotor de la salud física, doctor en Educación, UNED. 
actividad física de moderada a vigorosa intensidad (AFMV) que en áreas semiurbanas. Con respecto al contexto de la lección, se encontró que un tercio se dedicó a asuntos administrativos y un cuarto a la instrucción y aptitud física. En conclusión, la AF durante las clases de EF en las escuelas está por debajo de las recomendaciones internacionales.

Palabras claves: SOFIT, educación física, educación primaria.

\begin{abstract}
The purpose of the investigation was to describe the levels of physical activity (PA) of students of elementary schools in urban and semi-urban areas during physical education classes (PE), the context of the lesson, and teacher interactions. This was a cross-sectional observationaldescriptive study of 360 students during physical education classes in first cycle in elementary schools. The System for Observing Fitness Instruction Time (SOFIT) was used to evaluate these variables. As a result, students perform less physical activity than the average recommended by the literature. Only $30.2 \%$ and $9 \%$ of PE lessons corresponded to activities of moderate (AMI) and vigorous (AV) intensity. Women had lower levels of PA than men, and schools in urban areas did not show more physical activity of moderate to vigorous intensity than in semi-urban areas. According to the context of the lesson, it was found that one third is devoted to administrative matters and a quarter to instruction and physical fitness. In conclusion, PA during PE classes in schools is below international recommendations.
\end{abstract}

Keywords: SOFIT, physical education, primary education.

\title{
Introducción
}

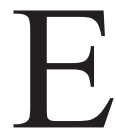
1 incremento en los niveles de sedentarismo, la falta de oportunidades de actividad física y el incremento de los niños con sobrepeso y obesidad en todo el mundo (Timmons et al., 2012) han llevado a considerar las escuelas como el escenario fundamental para que aquellos puedan ser activos y construyan las destrezas necesarias para enfrentar una cultura sedentaria. El enfocarse en las escuelas para 
facilitar la actividad física es una de las estrategias que ofrecen el mayor potencial para el desarrollo de los hábitos de movimiento, los cuales se puedan trasladar a la vida adulta (Bassett et al., 2013).

La promoción de la salud, la prevención de la obesidad y del sedentarismo no son solo un asunto del sistema de salud, son una responsabilidad de todos y las escuelas, a través de la educación física, desempeñan un rol determinante, en tanto ofrezcan las posibilidades para que los niños desarrollen conductas saludables de por vida. Sin embargo, pareciera que la meta de realizar una hora diaria de actividad física de moderada a vigorosa intensidad no se cumple; es aquí donde las instancias educativas han asumido el reto de darle respuesta a la pandemia del sedentarismo y la obesidad infantil, mediante una propuesta curricular en EF que promueva una cultura de movimiento desde la niñez (Ministerio de Educación Pública [MEP], 2013).

Las clases de educación física ofrecen el enlace esencial para que los niños logren alcanzar niveles de actividad física que los enrumben hacia el cumplimiento de las recomendaciones de realizar una hora diaria de $\mathrm{AF}$, lo cual les otorgará los beneficios en la prevención de enfermedades cardiodegenerativas, así como un mejor funcionamiento cognitivo, social y emocional (Hallal et al., 2012). No obstante, las clases de EF podrían no estar ofreciendo los resultados para la promoción de la actividad física escolar que se ha asumido por años (Bassett et al., 2013). Investigaciones efectuadas en otros entornos han reportado que los estudiantes típicamente se involucran de un $27 \%$ a un $47 \%$ en la lección de educación física (Springer et al., 2013), con actividad moderada. En una revisión sistemática de 40 estudios reportada por Sutherland et al. (2013), en el contexto de la educación primaria, se concluyó que los estudiantes se involucran solo en un $30 \%$ promedio en actividad moderada, durante el tiempo de la clase de EF.

Con respecto a la participación de los niños en la clase de EF, se ha reportado que, cuando este tipo de clases fue mixto, los niveles de actividad física y tiempo de juego fueron iguales para todos los alumnos, por lo que se invirtió menor cantidad de tiempo de la clase en asuntos administrativos (McKenzie et al., 2006); sin embargo, se recalca que en el nivel de tercer grado los niños fueron más activos que las niñas, durante el período de actividad libre al final de la clase.

El promover estilos de vida en movimiento en los estudiantes es un objetivo clave de los planes de estudio de educación física del 
Ministerio de Educación (MEP, 2013). Para lograr esto, las clases de educación física han sido estructuradas para que los alumnos reciban suficiente tiempo direccionado al desarrollo de habilidades y condición física, mientras reducen el lapso empleado en asuntos administrativos de la clase, junto con una mediación pedagógica del docente en la promoción de estilos de vida activos. En esta nueva propuesta programática, se ha reconocido a los educadores físicos como los docentes del sistema educativo que pueden proporcionar e impactar a sus estudiantes con estilos de vida activos, a través de las diferentes estrategias de apoyo social, pero, sobre todo, promoviendo la alfabetización en la realización de actividad física de por vida (Lox, Martin y Petruzzello, 2006).

En el contexto costarricense, la cantidad de la actividad física que los estudiantes realizan durante la clase de EF y el tipo administración de la clase, al grado en que los profesores de EF promueven estilos de vida activos, como se propone en los nuevos programas de educación primaria (MEP, 2013), son desconocidos, por lo que se hace necesario valorar estas variables, con el fin de medir el aporte de la nueva propuesta educativa de los programas de I y II ciclo, en la promoción de dichos estilos de vida.

Dada la carente evidencia en el contexto costarricense acerca de la calidad de las clases de educación física en el camino de la promoción de estilos de vida en movimiento, este estudio analizó el porcentaje del tiempo de la clase de EF dedicado a participar en actividades de moderadas a vigorosas de estudiantes, el contexto de la lección, la interacción del profesor con los alumnos durante la clase de educación física y las diferencias en relación con el género, en el marco de los nuevos programas de EF del Ministerio de Educación.

\section{Metodología}

\section{Participantes}

La muestra contempló la observación de niños y niñas $(n=360)$ con edades entre los 7 y los 9 años, de I ciclo, durante la clase de educación física de 6 escuelas públicas de la provincia de Heredia, seleccionadas al azar. En cada ciclo de cada escuela, se seleccionaron por conveniencia los grupos por observar, así como los estudiantes elegidos para ser observados, siguiendo el protocolo del SOFIT. 


\section{Variables}

Las variables dependientes de la intensidad de actividad física durante la clase, el contexto de instrucción y la promoción de la actividad física por parte del profesor fueron evaluadas, para lo cual se aplicó el instrumento de observación (SOFIT).

Los observadores de este estudio recibieron un entrenamiento inicial, que incluyó contemplar el video SOFIT 93 desarrollado por McKenzie (2009), seguido de prácticas de campo en diferentes centros educativos.

\section{Estrategia para minimizar el sesgo}

Cada período de observación usando el instrumento SOFIT, fue realizado por 2 observadores, para garantizar la calidad de las observaciones. Adicionalmente, durante el tiempo de la ejecución de las observaciones, se estableció la confiabilidad entre los observadores y si el resultado fue menor al $80 \%$, se repitió el proceso de entrenamiento.

\section{Instrumento por utilizar}

Se utilizó el instrumento SOFIT (Sistema para Observar la Instrucción de la Aptitud Física), validado por Rowe, Schuldheisz y van der Mars (1997), con el fin corroborar los niveles de actividad física de los niños. Previas a la aplicación del SOFIT, se realizaron observaciones de las áreas de instrucción en educación física, con el afán de verificar y ajustar, de ser necesario, el SOFIT. Todo lo anterior se llevó a cabo en el marco del protocolo de este instrumento (Mckenzie, 2012). La intensidad de la actividad física durante las clases de educación física, el contexto de la clase y el tipo de refuerzo del educador en la promoción de tal actividad, se realizó eligiendo al azar a 4 estudiantes de cada clase de educación física ( 2 hombres y 2 mujeres), quienes se observaron en secuencia rotatoria de 12 intervalos durante 20 segundos cada uno, repitiéndose las observaciones durante toda la clase y siguiendo un audio que indicó los tiempos de registro de la actividad. Con el propósito de determinar la intensidad de la actividad física en la clase de educación física, se usaron códigos para clasificar los niveles; este procedimiento se clasificó en 5 códigos: 1) acostado, 2) sentado, 3) parado, 4) caminando y 5) muy activo (corresponde a correr o una actividad con un gasto energético mayor). Estos códigos han sido calibrados monitoreando los latidos del corazón y el sistema ha sido validado en clases de educación física usando acelerómetros Caltrac (Sharma, Lee y Chung, 
2011). A partir de la cuantificación de dichos códigos, se estableció el índice de actividad física de moderada a vigorosa (IAFMV), sumando, porcentualmente, los códigos 4) caminando y 5) muy activo, del total del tiempo de la clase de educación física y del recreo.

Simultáneamente, con SOFIT se evaluó el contexto de la clase, el cual dirige el profesor de educación física. En este apartado, se identifican 7 variables que se codifican de la siguiente manera: $M$ ) contenido general, P) conocimiento específico, K) conocimiento general, F) acondicionamiento físico, S) desarrollo de habilidades, G) juego y O) otros. Finalmente, este instrumento de observación codifica el compromiso del docente durante la clase. La acción del docente es clasificada de 1 a 6 categorías. La primera categoría de acciones promueve aptitud física (P), está directamente relacionada con el nivel de involucramiento del alumno en actividades de aptitud física y es codificada cuando el docente incita o provee consecuencias para los alumnos acerca del compromiso de estos con la aptitud física. La segunda categoría demuestra aptitud física (D), identifica el momento cuando el docente sirve como modelo de compromiso con la aptitud física. Las cuatro categorías restantes (enseñanza general (I), gestión $(\mathrm{M})$, observa $(\mathrm{O})$ y otras tareas (T)) están indirectamente relacionadas con las oportunidades de realizar actividad física por los estudiantes.

\section{Procedimiento}

Una vez que el estudio fue aprobado por el Ministerio de Educación y se contó con la anuencia de los directores de las instituciones educativas, se obtuvo el consentimiento de los padres de los participantes. Las clases de educación física se escogieron por conveniencia por la orientadora de la institución, con el requisito de que fueran de primer ciclo, en las clases de la mañana y que las observaciones se realizaran sin aviso previo. Siguiendo el protocolo, los observadores llegaron 15 minutos antes del comienzo de la clase y ejecutaron la observación como fue descrita en la sección de instrumentos. Al finalizar la observación de la clase escogida, se llenó la hoja resumen de SOFIT, según se indica en el protocolo. 


\section{Análisis de datos}

Se realizó la estadística descriptiva calculando los promedios y las desviaciones estándar de las variables dependientes de actividad física, contexto de clase y promoción de la actividad física. Por medio de la prueba t-student de una muestra contra la población, se determinó si hubo diferencias significativas entre el promedio de actividad física de moderada a vigorosa de este estudio y la recomendación de dedicar al menos un $50 \%$ de la lección de EF a actividades de moderada a vigorosa intensidad, dada por instancias internacionales (Centers for Disease Control and Prevention [CDC], 2010). Para determinar si hubo diferencias significativas entre la actividad física moderada y las variables independientes de género y localización, se ejecutó una ANOVA factorial 2 × 2; al no encontrarse diferencias significativas, no se ejecutó la prueba post-hoc. Toda la información fue analizada mediante el Paquete Estadístico para las Ciencias Sociales (SPSS), versión 22.0 para Windows.

\section{Resultados}

Un total de 360 niños fueron observados durante la clase de educación física de I ciclo de 3 escuelas de la zona semiurbana y 3 públicas de la zona urbana de la provincia de Heredia. 
Tabla 1.

Promedio y desviaciones estándar en la proporción del tiempo de clase gastado en diferentes niveles de actividad física, contexto de clase e interacción del profesor

\begin{tabular}{|l|c|}
\hline \multicolumn{1}{|c|}{ Categoría } & Promedio/SD \\
\hline Actividad física moderada y alta & $39.2 \pm 14.5$ \\
\hline Acostado & $0.40 \pm 1.35$ \\
\hline Sentado & $28.2 \pm 16.1$ \\
\hline Parado & $32.2 \pm 14.8$ \\
\hline Caminando & $30.2 \pm 11.7$ \\
\hline Muy activo & $9.00 \pm 6.20$ \\
\hline Administración & $27.4 \pm 11.9$ \\
\hline Conocimiento & $13.0 \pm 10.2$ \\
\hline Aptitud física & $12.0 \pm 18.4$ \\
\hline Destrezas & $13.1 \pm 18.6$ \\
\hline Juego (libre) & $34.5 \pm 36.3$ \\
\hline Promoción de AF dentro de la clase & $28.6 \pm 13.3$ \\
\hline Promoción de AF fuera de la clase & $0.30 \pm 0.80$ \\
\hline Sin promoción & $71.2 \pm 13.5$ \\
\hline
\end{tabular}

La tabla 1 muestra los promedios y desviaciones estándar de tiempo lectivo para cada una de las variables evaluadas. En general, el $30.2 \%$ de la lección de educación física fue utilizada en actividad física de moderada intensidad y el $9 \%$ se gastó en actividad física de alta intensidad. De las lecciones observadas, el $39.2 \%$ se invirtieron en actividad física de moderada a vigorosa intensidad (AFMV). Casi un 25 $\%$ del tiempo de las lecciones se utilizó en actividades de aptitud física y la práctica de destrezas. En el contexto de la lección de educación física, un $27.4 \%$ fue dedicado a aspectos administrativos y un $34.5 \%$ a juego libre o supervisado. Con respecto a la interacción del profesor, en la promoción de la actividad física se obtuvo un $28.6 \%$. Las lecciones en escuelas ubicadas en regiones urbanas se caracterizaron por tener más tiempo en actividades de moderada intensidad (42.9\% frente a $37.0 \%$ ) y en actividad vigorosa (10.7\% frente a 7.8\%), en comparación con las áreas semiurbanas. En las lecciones de escuelas urbanas, se pasó más tiempo en actividades de acondicionamiento físico en comparación con las escuelas semiurbanas $(17.0 \%$ frente a $8.6 \%$,). En relación con los niveles de actividad física entre niños y niñas, se observó menor 
involucramiento de las niñas en las actividades de tiempo libre (36.28 $\%)$ en comparación con los niños (40.38\%), siendo similares en el resto de las observaciones de las lecciones de EF.

En cuanto al contexto de clase, la mayoría de tiempo de esta fue invertido en juego libre (34.5\%). El tiempo empleado en práctica de destrezas fue de $13.1 \%$, que, junto al lapso dedicado a la aptitud física de (12.0\%), representa un cuarto de tiempo del total de la clase. En el ámbito de la interacción del profesor y la promoción de actividad física, el tiempo que los docentes utilizaron en animar y estimular conductas activas en clases fue de un tercio de las clases $(28.6 \%$ ) y de un $0.30 \%$ fuera de ellas. Como era esperado, el porcentaje de actividad más bajo observado ocurrió durante los períodos en los que el profesor transmitió información y mientras transcurría el tiempo de administración de la clase; primariamente, a lo largo de este, los estudiantes estuvieron sentados o de pie (40.4\%).

Por otro lado, la figura 1 compara el porcentaje de actividad física que los estudiantes de este estudio invirtieron en actividad física de moderada a vigorosa, durante la observación (39.2 \%), en relación con las recomendaciones mundiales del tiempo que los estudiantes deben invertir en este tipo de actividad (50\%).

\section{Figura 1}

Comparación de actividad física (moderada-vigorosa) observada con las recomendaciones de la CDC (2010)

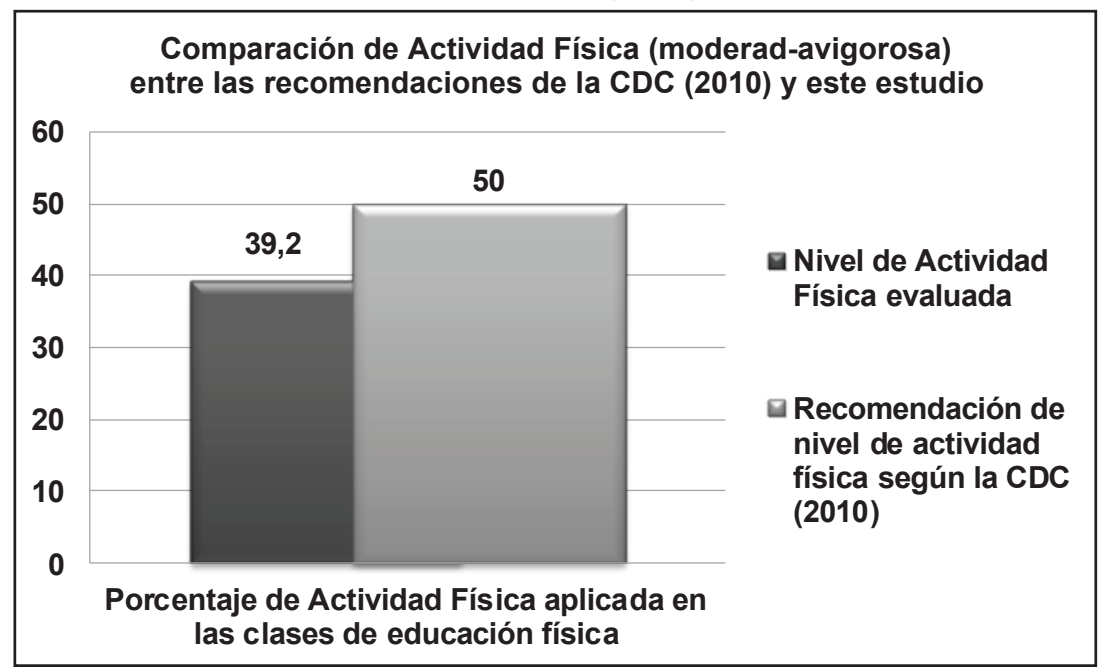


Por medio de la prueba t-student de una muestra contra la población, se encontraron diferencias significativas $(\mathrm{p}<0.05)$ en el promedio de los puntajes obtenidos de actividad física moderada de los estudiantes en las clases de EF $(M=39.2)$ y el tiempo apropiado de actividad física de los estudiantes recomendado por la literatura $(\mathrm{M}=50)$. Los estudiantes de esta muestra realizan menos actividad física que el promedio recomendado por la literatura.

Por medio de la prueba de ANOVA 2 X 2, se encontró diferencias significativas en relación con el género $(p<0.000)$ sobre el promedio de actividad física de los alumnos durante la clase de EF; independientemente, el género causó una diferencia significativa sobre la cantidad de actividad física que realizaron los participantes en este estudio, no así sucedió con la zona de procedencia urbana o semiurbana de los colaboradores. Debido a los resultados anteriores, no se presentaron interacciones entre las variables de género y escuela de procedencia. Esta falta de interacción podría haber sido diferente, si se hubieran escogido escuelas de zonas rurales, debido a las condiciones diferenciadas de ambos entornos (Álvarez, 2015).

\section{Discusión}

\section{Niveles de actividad física en las clases de educación física}

Este es uno de los primeros estudios que describen los niveles de actividad física, el contexto de la lección y las interacciones del maestro durante las clases de educación física en escuelas públicas costarricenses, lo cual se hace indispensable, con el fin de entender la contribución de estas clases en la promoción de estilos de vida activos. Lo anterior en las áreas específicas de cantidad de actividad física durante la clase, organización de esta y el apoyo social del educador, como mediadores en la construcción de dichos estilos de vida activos en los educandos.

Los hallazgos de este estudio muestran que los estudiantes gastaron un promedio de $30.2 \%$ de la lección de educación física en AFM y menos del $10 \%$ en AV. Aproximadamente el $30 \%$ del tiempo de la lección se dedicó a asuntos administrativos, mientras la promoción de la actividad física en el contexto de clase fue inexistente. En aras de paliar el porcentaje tan alto dedicado a asuntos administrativos, se podría integrar los momentos de entrega de la información a los del calentamiento. 
Los hallazgos de este estudio coinciden con investigaciones previas (Fairclough y Stratton, 2005; Sutherland et al., 2013) que reportaron que los estudiantes de primaria, típicamente, solo llegan de un $27 \%$ a un $47 \%$ del tiempo de clase dedicado a actividades de moderada a alta intensidad. Sin embargo, cuando se comparan los resultados con las recomendaciones internacionales, respecto al $50 \%$ de la lección de educación física dedicada a realizar AFMV intensidad (CDC, 2010), los resultados de este estudio están lejos de la meta y todavía es más evidente la brecha, cuando utilizamos el criterio de $80 \%$ de la clase de educación física dedicada a AFMV, ambas sugerencias dadas por las autoridades educativas costarricenses en los nuevos programas de EF (MEP, 2013).

Contrario al estudio de Sutherland et al. (2013), las escuelas ubicadas en áreas urbanas tuvieron lecciones de EF significativamente más activas que las localizadas en áreas semiurbanas. Aunque los niveles de AFMV en las escuelas urbanas en este estudio fueron porcentualmente mayores que en las semiurbanas, estas no resultan significativas y en ninguno de los casos se llega al $50 \%$ del tiempo de lección recomendado en AFMV (CDC, 2010). Los resultados de este estudio fueron consistentes con los datos de una revisión sistemática de escuelas intermedias y secundarias, los cuales mostraron que, cuando se utilizaron dos métodos de evaluación para determinar la intensidad de la actividad física, el $40 \%$ del tiempo de lección de educación física, se gastó en AFMV (Fairclough y Stratton, 2005).

La indagación actual encontró que los estudiantes pasaron más tiempo sentados y de pie y menos tiempo en AFM que otros estudios que analizaron los niveles de actividad física en las lecciones de educación física (Dudley, Okely, Pearson, Cotton y Caputi, 2012a; Brown y Holland, 2005). A diferencia de esta investigación, que incluyó clases mixtas, el estudio realizado por Dudley, Okely, Pearson, Cotton y Caputi (2012b), en el cual solo participaron niños, reportó niveles más altos de actividad física, hecho que podría haber aumentado el promedio de AFMV en ese trabajo.

Los niveles de AFMV observados en este estudio pueden sobreestimar los niveles de actividad física en educación física, ya que la AFMV varía dependiendo del método de evaluación. Los estudios que comparan directamente los métodos han demostrado que las técnicas observacionales tienden a mostrar que la MVPA es consistentemente 
más alta que la MVPA evaluada por el acelerómetro (McClain, Abraham, Brosseau y Tudor-Lock, 2008). Esto ocurre con observaciones usando SOFIT, que incluyen el caminar como una actividad de AFMV, de baja intensidad. Las lecciones de educación física pueden ser una de las pocas oportunidades que los estudiantes tienen para dedicarse a la actividad física y a adquirir destrezas conductuales, para ser activos de por vida. Por ello, los niveles bajos de APMV en las lecciones de educación física representan un elemento propicio para ser intervenido, con el fin de lograr mayores niveles de actividad física.

Se ha reportado que, cuando las clases de EF eran mixtas, los niveles de actividad física y tiempo de juego fueron iguales para todos los alumnos, presentándose menor cantidad de tiempo de la clase en asuntos administrativos (McKenzie et al., 2006). Sin embargo, los hallazgos de este estudio mostraron que las niñas realizaron menor cantidad de actividad física durante las clases de $\mathrm{EF}$, lo cual quedó evidenciado durante el período de actividad libre, en el que las niñas se aglutinaron a hablar.

En el caso de las mujeres, es reconocido que factores biológicos, ambientales y sociales las predisponen a ser sedentarias y a enfrentar enfermedades cardiodegenerativas, osteoporosis, diabetes, obesidad, tanto como desórdenes alimenticios, situaciones que tienen su inicio a edades tempranas (Shephard y Trudeau, 2000). Esta situación es especialmente preocupante en el caso de las niñas sedentarias, por el hecho de que ellas tendrán una posibilidad mayor de ser inactivas en su vida futura.

\section{Contexto de la clase}

A diferencia de los momentos de la lección de educación física dados en los nuevos programas de educación física, en el nivel nacional (MEP, 2013), los resultados de esta investigación ofrecen tres que se marcan claramente en las observaciones: uno administrativo, que significó casi $27 \%$ de la duración de la clase; otro de actividades de actitud física e instrucción, con un final de juego, y uno de actividad física, con un $34 \%$ del tiempo de clase.

Un hallazgo interesante en este estudio es la gran proporción del tiempo de las clases de EF (cerca $30 \%$ ), que se invirtió en asuntos administrativos. Las indagaciones han informado que la gestión de la labor administrativa suele variar entre el $15 \%$ y el $26 \%$ del tiempo lectivo (Jago et al., 2009). El alto porcentaje de tiempo dedicado a la 
gestión puede ser común en las clases de educación física en Costa Rica; sin embargo, se ha reportado que los profesores de las escuelas de atención prioritaria o en zonas urbano-marginales podrían requerir más lapso de gestión, debido a problemas de disciplina en el aula (Hemphill et al., 2010). La actividad física es más baja durante las actividades administrativas y de gestión, por lo que se necesita desarrollar estrategias para disminuir estos periodos (Skala et al., 2012), enfocándose en el desarrollo de destrezas organizativas de la clase, en el manejo, la instrucción y utilización de recursos que promuevan más actividades de moderada a alta intensidad, lo que se considera que podría incrementar hasta en un $24 \%$ la actividad de moderada a alta intensidad (Lonsdale et al., 2013).

La enseñanza de destrezas representó un $13 \%$ del tiempo lectivo, más alta que la observada en otros estudios (Dudley et al., 2012b). Sin embargo, debido a que el desarrollo de las habilidades motoras se ha asociado con una mayor cantidad de tiempo de práctica de la actividad física, parece particularmente importante, desde un punto de vista pedagógico y de salud pública, asegurar que a este aspecto del contexto de las lecciones se le dé un mayor énfasis. Hay evidencia que sugiere que, a menos que las destrezas básicas motoras sean aprendidas durante las etapas iniciales escolares, sería poco probable que los niños adquieran altos niveles de competencia (Gallahue y Ozmun, 2006); lo que podría explicar la dificultad que enfrentan los educadores físicos a la hora de involucrar a los estudiantes de secundaria en actividad física (Álvarez, 2016).

En este estudio, se reportó que un $13 \%$ de la clase de educación física fue dedicado a aspectos de instrucción verbal del conocimiento, lo cual, junto con el tiempo administrativo, brinda un $43 \%$ de actividad sedentaria dentro de la clase. Aunque hallazgos similares se han reportado (Bevans, Fitzpatrick, Sánchez, Riley y Forrest, 2010), la recomendación para paliar tal situación es la de integrar los periodos de instrucción verbal a una dinámica más marcada en el uso de la exploración del movimiento, mediante la que la información pueda ser dada en espacios como el estiramiento o los períodos de relajamiento. Como un aspecto esperado, se detectó cantidades de tiempo sustanciales en la transición entre actividades, especialmente, cuando los estudiantes se cambiaron o se desplazaron a otras actividades. El reducir los espacios de transición podría ser una gran oportunidad para ofrecer alternativas de movimiento. 


\section{Promoción de la actividad física}

Los resultados de este estudio mostraron que en un $71 \%$ de las lecciones observadas los docentes no promovieron conductas activas; por el contrario, ellos evidenciaron una relación mecánica y fría, a la hora de promocionar la actividad física entre los alumnos. Aunque en los nuevos programas de educación física se le asigna un rol determinante al docente en la promoción de estilos de vida activos, a través de una mediación pedagógica (MEP, 2013), no existen guías o recomendaciones de lo que se espera en el contexto de clases de parte del educador. En la línea del docente como modelo, los diferentes marcos de referencia teóricos han enfatizado su labor determinante en la educación física, como ente generador de conductas activas, a través de apoyos instrumentales, modelaje directo y apoyo emocional (Lox, Martin y Petruzzello, 2006; MEP, 2013).

La promoción del docente hacia conductas activas incluye el dar instrucciones, alentar o animar verbalmente comportamientos activos en los alumnos, el demostrar una actividad o una destreza, la participación directa con los estudiantes y el propiciar el desarrollo de destrezas conductuales. Este estudio demostró que los educadores físicos participaron verbalmente un $28.6 \%$ de la clase dando instrucciones. En una investigación previa internacional, se mostró que la participación del docente de educación física promoviendo conductas activas fue de un $30.8 \%$ del tiempo de clase (Dudley et al., 2012a). Los resultados tan bajos obtenidos en este estudio, en comparación con otros, se podrían explicar por el gran énfasis que las clases de educación física observadas le asignan al espacio de actividad libre supervisado (34.5\%).

Consistente con las investigaciones llevadas a cabo en escuelas de secundaria (McKenzie, 2006; Dudley, Okely, Pearson, Cotton y Caputi, 2012a), este trabajo encontró que menos de un $1 \%$ fue dedicado a promover actividad física fuera de la clase, lo que podría estar menoscabando uno de los objetivos principales de la educación física (Organización de la Naciones Unidas [UNESCO], 2015), que es el brindar conocimientos, habilidades y actitudes que permitan a los estudiantes desarrollar estilos de vida activos, durante toda la vida (Lonsdale et al., 2013). La inclusión de estrategias comunitarias, como la vinculación con organizaciones deportivas, en las intervenciones de actividad física escolar, podría complementar y reforzar el mandato más amplio de la educación física para inculcar la actividad de toda la vida. 
En concordancia con los resultados de la labor del educador físico como promotor de la actividad física (MEP, 2013) y asumiendo los retos de las recomendaciones de dicho tipo de actividad en la niñez (CDC, 2010), existe el desafío de asumir la educación física no solo como un momento de instrucción de destrezas, sino también como el espacio ideal para promover actividad física de por vida (Heidorn y Welch, 2010), en el que los estudiantes adquieran alfabetización en los estilos de vida saludables. El impacto de la educación física debería no ser limitado al entorno de la clase y estrategias adicionales deberían tomarse para promover el movimiento más allá de ella. Autores como Casey (2014) y Ward et al. (2007) recomiendan que los programas y la formación de profesionales en movimiento incluya las destrezas conductuales, sociales y físicas para que los alumnos se involucren de por vida en actividad física.

Aunque no fue un objetivo de este estudio comparar la experiencia de los docentes, se ha mostrado que los maestros más experimentados realizaron clases con más MVPA y AV que los maestros menos experimentados. Además, los profesores realizaron lecciones con un número significativamente mayor de AVA que las profesoras. Esto contrasta con otros estudios de investigación clásicos (McKenzie et al., 1995; McKenzie et al., 2000), en los cuales no se reportaron diferencias, lo que sugiere que los factores modificables deberían considerarse cuando se diseñen programas para incrementar la actividad física de los alumnos.

\section{Conclusiones}

Aunque se reconoce que el entorno de las lecciones de educación física ofrece el potencial de promover conductas activas de por vida, la AF durante las clases de EF en las escuelas observadas está por debajo de las recomendaciones internacionales, siendo más activos los niños que las niñas. En el área del contexto de la clase, se encontró que casi un tercio de la lección es dedicado a asuntos administrativos y un 34 $\%$ a actividad libre, con solo un $28.6 \%$ utilizado en promoción de la actividad física por parte del profesor. Este estudio identifica áreas específicas que deberían ser focalizadas en el proceso de mejoramiento de la administración de la clase de educación física, en el marco de los nuevos programas. Se reconoce que la muestra de escuelas no representa el gran espectro de las existentes en el nivel nacional, pero sí 
evidencia una posibilidad de establecer puntos de inicio en el mejoramiento e impacto de las lecciones de educación física en los años que el desarrollo de estilos de vida en movimiento son determinantes.

\section{Referencias}

Álvarez, C. (2015). Factores que influencian un estilo de vida sedentario en las estudiantes de cuarto ciclo de los colegios públicos. MHSALUD: Revista en Ciencias del Movimiento Humano y Salud, 12(1), 1-17. Doi: 10.15359/mhs.12-1.1

Álvarez, C. (2016). Entendiendo los factores que determinan la actividad física en el entorno escolar desde la perspectiva de los niños y niñas. MHSALUD: Revista en Ciencias del Movimiento Humano y Salud, 13(1), 1-17. Doi: 10.15359/mhs.13-1.2

Bassett, D. R., Fitzhugh, E. C., Heath, G. W., Erwin, P. C., Frederick, G. M., Wolff, D. L., Welch, W. A. y Stout, A. B. (2013). Estimated energy expenditures for school-based policies and active living. American Journal of Preventive Medicine, 44(2), 108-113. Doi: 10.1016/j.amepre.2012.10.017

Bevans, K., Fitzpatrick, L., Sánchez, A., Riley, A. W. y Forrest, C. (2010). Physical education resources, class management, and student physical activity levels: a structure-process-outcome approach to evaluating physical education effectiveness. Journal of School Health, 80(12), 573-580. Doi: 10.1111/j.1746-1561.2010.00544.x

Brown, T. D. y Holland, B. V. (2005). Student physical activity and lesson context during physical education. ACHPER Healthy Lifestyles Journal, 52(3/4), 17-23. Recuperado de https://www. cabdirect.org/cabdirect/abstract/20053225052

Casey, A. (2014). Models-based practice: great white hope or white elephant? Physical Education and Sport Pedagogy, 19(1), 1834. Recuperado de https://dspace.lboro.ac.uk/dspace-jspui/bitstream/2134/19544/4/PESP\%20Casey\%202014.pdf

Centers for Disease Control and Prevention [CDC]. (2010). Strategies to improve the quality of physical education. En Services US Department of Health and Human Services (editor), Atlanta, Services US Department of Health and Human Services. Recuperado de https://www.cdc.gov/healthyschools/pecat/quality_pe.pdf 
Análisis de los niveles de actividad física de los estudiantes, el tipo de contenidos de la lección y la interacción del profesor durante la clase de educación física en escuelas públicas

Dudley, D., Okely, A., Pearson, P., Cotton, W. y Caputi., P. (2012a). Changes in physical activity levels, lesson context, and teacher interaction during physical education in culturally and linguistically diverse Australian school. International Journal of Behavioral Nutrition and Physical Activity, 9(114), 2-9. Doi: 10.1186/1479-5868-9-114

Dudley, D., Okely, A., Pearson, P., Cotton, W. y Caputi., P. (2012b). Physical activity levels and movement skill instruction in secondary school physical education. Journal of Science and Medicine in Sport, 15(3), 231-237. Doi: 10.1016/j.jsams.2011.10.005

Fairclough, S. y Stratton, G. (2005). Physical activity levels in middle and high school physical education: A review. Human Kinetics Journals, 17(3), 217-236. Doi: 10.1123/pes.17.3.217

Gallahue D. L. y Ozmun, J. C. (2006). Understanding motor development: Infants, children, adolescents, adults. $6 .{ }^{\mathrm{a}}$ edición. Boston: McGraw-Hill.

Hallal, P. C., Andersen, L. B., Bull F. C., Guthlod, R., Haskell, W., Ekelund, U y Lancet Physical Activity Series Working Group. (2012). Global physical activity levels: surveillance progress, pitfalls, and prospects. The Lancet, 380(9838), 247-257. Doi: 10.1016/ S0140-6736(12)60646-1

Heidorn, B. y Welch, M. M. (2010). Teaching affective qualities in physical education. Strategies, 23(5), 16-21. Recuperado de https:// www.researchgate.net/publication/233522478_Teaching_Affective_Qualities_in_Physical_Education

Hemphill, S. A., Toumbourou, J. W., Smith, R., Kendall, G. E., Rowland, B., Freiberg, K. y Williams, J. W. (2010). Are rates of school suspension higher in socially disadvantaged neighbourhoods? An Australian study. Health Promotion Journal Australian, 21(1),12-18. Recuperado de https://pdfs.semanticscholar.org/ ede8/2f3c401732cb8b55f3fa8fdd15e58ce8a94e.pdf

Jago, R., McMurray, R. G., Bassin, S., Pyle, L., Bruecker, S., Jakicic, J. M., Moe, E., Murray, T. y Volpe, S. L. (2009). Modifying middle school physical education: piloting strategies to increase physical activity. Pediatric Exercise Science, 21(2), 171-185. Recuperado de https://www.ncbi.nlm.nih.gov/pmc/articles/PMC2705879/

Lonsdale, C., Rosenkranz, R. R., Peralta, L. R., Bennie, A., Fahey, P. y Lubans D. R. (2013). A systematic review and meta-analysis of 
interventions designed to increase moderate-to-vigorous physical activity in school physical education lessons. Preventive Medicine Journal, 56(2),152-161. Doi: 10.1016/j.ypmed.2012.12.004

Lox, C., Martin, K. y Petruzzello, S. (2006). The Psychology of Exercise: Integrating Theory and Practice. $4 .^{a}$ edición. Arizona, Estados Unidos: Holcomb Hathaway Publishers.

McClain, J., Abraham, T., Brusseau, T. A. y Tudor-Locke, C. (2008). Epoch length and accelerometer out-puts in children: comparison to direct observation. Medicine \& Science in Sports \& Exercise, 40(12), 2080-2087. Doi: 10.1249/MSS.0b013e3181824d98

McKenzie, T. L., Catellier, D. J., Conway, T., Lytle, L. A., Grieser, M., Webber, L. A., Pratt, C. y Elder, J. (2006). Girls' activity levels and lesson contexts in middle school PE: TAAG Baseline. Medicine \& Science in Sports \& Exercise, 38(7),1229-1235. Doi: 10.1249/01.mss.0000227307.34149.f3

McKenzie, T. L. (2009). Seeing is believing: Observing physical activity and its contexts. Research Quarterly for Exercise and Sport, 81(2), 113-122. Recuperado de http://thomckenzie.com/ useful-tools/observation/

McKenzie, T. L. (2012). SOFIT: System for observing fitness instruction time. Overview and training manual. Recuperado de https://activelivingresearch.org/sites/default/files/SOFIT_Protocols_05.01.15.pdf

McKenzie, T. L., Marshall, S., Sallis, J. y Conway, T. (2000). Student activity levels, lesson context, and teacher behavior during middle school physical education. Research Quarterly for Exercise and Sport, 71(3), 249-259. Doi: 10.1080/02701367.2000.10608905

McKenzie, T. L., Feldman, H., Woods, S. E., Romero, K. A., Dahlstrom, V., Stone, E. J., Strikmiller, P. K., Williston, J. M. y Harsha, D. W. (1995). Children's activity levels and lesson context during thirdgrade physical education. Research Quarterly for Exercise and Sport, 66(3), 184-193. Doi: 10.1080/02701367.1995.10608832

Ministerio de Educación Pública de Costa Rica [MEP]. (2013). Programas de Estudio de Educación Física. San José, Costa Rica: MEP. Recuperado de http://www.mep.go.cr/sites/default/files/descargas/programas-de-estudio/educfisica3cicloydiversificada.pdf Organización de la Naciones Unidas [UNESCO]. (2015). Educación Física de Calidad (EFC). Guía para los responsables políticos. 
Análisis de los niveles de actividad física de los estudiantes, el tipo de contenidos de la lección y la interacción del profesor durante la clase de educación física en escuelas públicas

Paris, Francia. Recuperado de http://unesdoc.unesco.org/images/0023/002313/231340S.pdf

Rowe, P. J., Schuldheisz, J. M., van der Mars, H. (1997). Measuring physical activity in physical education: Validation of the SOFIT direct observation instrument for use with first to eighth grade students. Pediatric Exercise Science, 9(2), 136-149.

Sharma, A., Lee, Y. D. y Chung, W. Y. (2011). High accuracy human activity monitoring using neural network. En Convergence and Hybrid Information Technology. ICCIT'08. Third International Conference on 1, 430-435. Doi: 10.1109/ICCIT.2008.394

Shephard, R. J. y Trudeau, F. (2000). The legacy of physical education: Influences on adult lifestyle. Pediatric Exercise Science, 12(1), 34-50. Doi: 10.1123/pes.12.1.34

Skala, K. A., Springer, A. E., Sharma, S. V., Hoelscher, D. M. y Kelder, S. H. (2012). Environmental characteristics and student physical activity in PE class: findings from two large urban areas of Texas. Journal of Physical Activity and Health, 9(4), 481-491. Recuperado de https://www.ncbi.nlm.nih.gov/pmc/articles/PMC3245768/ Springer, A. E., Kelder, S. H., Byrd-Williams, C. E., Pasch, K. E., Ranjit, N., Delk, J. E., Hoelscher, D. M. (2013). Promoting energy-balance behaviors among ethnically diverse adolescents: overview and baseline findings of The Central Texas CATCH Middle School Project. Health Education \& Behavior, 40(5), 559-570. Doi: 10.1177/1090198112459516

Sutherland, R., Campbell, E., Lubans, D. R., Morgan, P. J., Okely, A. D., Nathan, N., Wolfenden, L., Jones, J., Davies, L., Gillham, K. y Wiggers, J. (2013). A cluster randomised trial of a school-based intervention to prevent decline in adolescent physical activity levels: study protocol for the 'Physical Activity 4 Everyone' trial. BMC Public Health, 13(1), 57. Doi: 10.1186/1471-2458-13-57

Timmons, B. W., Leblanc, A. G., Carson, V., Connor, S., Dillman, C., Janssen, I., Kho, M. E., Spence, J. C., Stearns, J. A. y Tremblay, M. S. (2012). Systematic review of physical activity and health in the early years (aged 0-4 years). Applied Physiology, Nutrition, and Metabolism, 37(4), 773-92. Doi:10.1139/h2012-070

Ward, D., Saunders, R. y Pate, R. (2007). Physical Activity Interventions in Children and Adolescents. USA: Human Kinetics Publishers. 
